\title{
Anti-inflammatory, Antibacterial, Toxicological Profile, and In Silico Studies of Dimeric Naphthoquinones from Diospyros lotus
}

\author{
Abdur Rauf $\mathbb{D}^{1},{ }^{1}$ Tareq Abu-Izneid $\left(\mathbb{D},{ }^{2}\right.$ Umer Rashid $\mathbb{D}^{3},{ }^{3}$ Fahad A. Alhumaydhi $\mathbb{D},{ }^{4}$ \\ Saud Bawazeer $\odot{ }^{5}$, Anees Ahmed Khalil $\odot{ }^{6}$ Abdullah S. M. Aljohani $\odot{ }^{7}$ \\ Emad M. Abdallah $\oplus,{ }^{8}$ Abdel Rahman Al-Tawaha $₫{ }^{9}$ Yahia Naseer Mabkhot $₫,{ }^{10,11}$ \\ Mohammad Ali Shariati $₫{ }^{12}$ Sergey Plygun $₫,{ }^{12,13,14}$ Md. Sahab Uddin $₫{ }^{15,16}$ \\ and Godswill Ntsomboh Ntsefong $\mathbb{D}^{17}$ \\ ${ }^{1}$ Department of Chemistry, University of Swabi, Swabi-Anbar-, 23430 KPK, Pakistan \\ ${ }^{2}$ Department of Pharmaceutical Sciences, College of Pharmacy, Al Ain University of Science and Technology, Al Ain Campus, UAE \\ ${ }^{3}$ Department of Chemistry, COMSATS University Islamabad, Abbottabad Campus, 22060 Abbottabad, Pakistan \\ ${ }^{4}$ Department of Medical Laboratories, College of Applied Medical Sciences, Qassim University, Buraydah, Saudi Arabia \\ ${ }^{5}$ Department of Pharmaceutical Chemistry, Faculty of Pharmacy, Umm Al-Qura University, Makkah, P.O. Box 42, Saudi Arabia \\ ${ }^{6}$ University Institute of Diet and Nutritional Sciences, Faculty of Allied Health Sciences, The University of Lahore, Pakistan \\ ${ }^{7}$ Department of Veterinary Medicine, College of Agriculture and Veterinary Medicine, Qassim University, Buraydah, Saudi Arabia \\ ${ }^{8}$ Department of Laboratory Sciences, College of Sciences and Arts at Al-Rass, Qassim University, Saudi Arabia \\ ${ }^{9}$ Department of Biological Sciences, Al-Hussein Bin Talal University, Maan, Jordan \\ ${ }^{10}$ Department of Pharmaceutical Chemistry, College of Pharmacy, King Khalid University, Abha, 61421, Saudi Arabia \\ ${ }^{11}$ Research Center for Advanced Materials Sciences (RCAMS), King Khalid University, 61413-Abha, 6113, Saudi Arabia \\ ${ }^{12}$ Laboratory of Biocontrol and Antimicrobial Resistance, Orel State University Named after I.S. Turgenev, 302026 Orel, Russia \\ ${ }^{13}$ European Society of Clinical Microbiology and Infectious Diseases, Basel 4051, Switzerland \\ ${ }^{14}$ All Russian Research Institute of Phytopathology, Moscow Region 143050, Russia \\ ${ }^{15}$ Department of Pharmacy, Southeast University, Dhaka, Bangladesh \\ ${ }^{16}$ Pharmakon Neuroscience Research Network, Dhaka, Bangladesh \\ ${ }^{17}$ Department of Plant Biology, Faculty of Science, \\ University of Yaounde $1 \&$ Institute of Agricultural Research for Development (IRAD), Cameroon
}

Correspondence should be addressed to Abdur Rauf; mashaljcs@yahoo.com

Received 1 March 2020; Accepted 7 May 2020; Published 27 May 2020

Academic Editor: Ercan Bursal

Copyright (c) 2020 Abdur Rauf et al. This is an open access article distributed under the Creative Commons Attribution License, which permits unrestricted use, distribution, and reproduction in any medium, provided the original work is properly cited.

Diospyros lotus, also known as date-plum, belongs to the Ebenaceae family and is mostly recognized as a rootstock for D. kaki. Similar classes of naphthoquinones in D. lotus are investigated against cancer and inflammation and have antimicrobial, sedative, and analgesic properties. Six chemical constituents (1-6) were isolated from Diospyros lotus and tested for antiinflammatory effects at the dose of 2.5 and $5 \mathrm{mg} / \mathrm{kg}$, i.p., using carrageenan (1\%, $0.05 \mathrm{ml})$-induced paw edema. The maximum protection against carrageenan-induced edema was observed for compounds 1 and 2 . Both studied compounds demonstrated significant anti-inflammatory effect after the $3^{\text {rd }}$ hour of posttreatment. The maximum anti-inflammatory effect of compound 1 was $85.96 \%$, while that of compound 2 was $81.44 \%$, followed by compounds 5 and 6 , which exhibited $80.11 \%$ and $82.45 \%$ effect, respectively. Similarly, histamine-induced inflammation was significantly antagonized by 1, 2, 5, and 6 with $87.99 \%, 82.18 \pm 1.8$, $80.40 \pm 1.59$, and $77.44 \%$ effects, respectively, at $5 \mathrm{mg} / \mathrm{kg}$ after the $2^{\text {nd }}$ hour of posttreatment. The rest of the tested compounds did not show any significant effect as compared to the negative control. Interestingly, no toxicity was observed at higher doses. Moreover, the extracted compounds showed remarkable antibacterial activity against the Gram-positive bacteria and no effect against the Gram-negative bacteria. Docking studies on target cyclooxygenases showed that all the compounds established interactions with the key amino acid residues present in the additional pocket of COX-2. Hence, these compounds may act as 
selective COX-2 inhibitors. In conclusion, the findings of the current study suggest that the roots of Diospyros lotus may contain some anti-inflammatory and antibacterial agents with minimal toxicological effects and accordingly this plant product is recommended for further investigations.

\section{Introduction}

The genus Diospyros comprises various species (nearly 760) and belongs to the family of flowering plants (shrubs and trees) known as Ebenaceae. This diverse genus is commonly available in the subtropical (Pakistan and India) and tropical (tropical America and Africa) regions around the globe [1]. Since ancient times, numerous species of Diospyros have been utilized as therapeutic agents in folk medications. Diospyros lotus, also known as date-plum, can achieve the height of up to 15 to 30 meters under optimum (semishaded) cultivation environment. Scientific investigations have reported different medicinal perspectives of various parts of $D$. lotus like analgesic (leaves), carminative (fruit), sedative (seeds), and febrifuge (bark) [2, 3].

Similarly, leaf extracts from Japanese persimmon (Diospyros kaki) along with jasmine have been employed in antitobacco toffees. Numerous triterpenes like ursane, oleanane, and lupine are efficiently isolated from plant cellular structures and have shown anti-inflammatory characteristics [2]. Traditionally, Diospyros species are used as therapeutic medicine for treatment of hiccups, bedwetting, insomnia, hypertension, dyspnea, pains (muscular and joints), intestinal worms, and fever $[4,5]$. Isolated bioactive compounds and extracts from different parts of $D$. lotus have also been reported to possess strong antiproliferative potentials [6].

It is evident from the already-published literature that quinone molecules are the main moieties in currently available drugs (saintopin, mitomycin, daunorubicin, anthracyclines, mitoxantrones, and doxorubicin) used for the treatment of cancers [7-9]. Additionally, few naphthoquinones (plumbagin) isolated from plant matrices of various Diospyros species have demonstrated significant potent cytotoxic characteristics [10-13]. Therefore, this study was designed to investigate the anti-inflammatory potential of six (1-6) di-naphthodiospyrols isolated from $D$. lotus roots.

\section{Materials and Methods}

2.1. Plant Material. In May 2009, roots of Diospyros lotus were brought from Razagram (Dir, Khyber Pakhtunkhwa), Pakistan. A voucher specimen (Bot. 20036 (PUP)) was placed in the Herbarium of the Botany Department at UOP, Pakistan, after authentication by Dr. Abdur Rashid (taxonomist).

2.2. Animals. In this experimentation, $\mathrm{BALB} / \mathrm{c}$ male mice were used to authenticate the anti-inflammatory potential of six isolated compounds from roots of $D$. lotus. These male mice were bought from National Institute of Health (NIH), Islamabad, Pakistan. In the animal room, they were kept at $25^{\circ} \mathrm{C}$ and maintained in light-dark cycle (12-12 hours) conditions along with ad libitum provision of water and normal food. All the experimental procedure received prior approval from the ethical committee of the Department of Pharmacy, University of Peshawar (UOP), KPK, Pakistan.

2.3. Extraction and Isolation. Initially, D. lotus roots weighing $14 \mathrm{~kg}$ were dried under shade and were converted to powder by using a commercial scale grinder. Powdered Diospyros lotus roots were repeatedly extracted at room temperature by placing them in methanol $(\mathrm{MeOH})$ solvent for 6 days along with periodic mixing through maceration. Collected extracts were pooled together and subjected to rotary concentration $\left(40 \pm 5^{\circ} \mathrm{C}\right)$ for evaporation of $\mathrm{MeOH}$ and concentration of extracts under vacuum conditions. This vacuum concentration resulted in the collection of a red residue (202 grams) which was suspended in water prior to its partitioning with four different solvents, i.e., $n$-butanol $(n-\mathrm{BuOH})$, ethyl acetate (EtOAc), chloroform $\left(\mathrm{CHCl}_{3}\right)$, and $n$-hexane $(n-\mathrm{hx})$, by following the procedures adopted by Padhyem et al. [8], Rauf et al. [9], and Bawazeer et al. $[10,11]$.

The chloroform fraction, i.e., F-1 (30 grams) was applied to column chromatography prepared from silica gel 60 (Merck- $5 \times 60 \mathrm{~cm})$ followed by elution with the gradient $n$-hexane-ethyl acetate $(100: 0$ to $0: 100)$ solvent system. On the basis of TLC profiling, 105 fractions, i.e., RF1 to RF105, were obtained. By combining RF1-RF10 (fractions), a new subfraction, i.e., SF1 (2 grams) was obtained and was again applied to column chromatography (C.C). This chromatographic system was eluted using $n$-hexane as eluting solvent to collect red color fatty acid residue. Depending upon TLC profile, RF11-RF105 (fractions) were pooled together to form SF3 (9.89 grams) and SF4 (9.89 grams) as two new major subfractions. Afterwards, SF4 (9.89 grams) was further subjected to C.C and was eluted through $n$-hexane-ethyl acetate $(100: 0$ to $100: 15)$. This resulted in the collection of sixty fractions which were again pooled depending upon TLC profile and generated major fractions (MF1: 5.44 grams; MF2: 3.41 grams). The major fraction (MF1) was applied on preparative TLC prepared from Merck silica gel $60\left(\mathrm{~F}_{254}\right.$, a florescence indicator). Moreover, this chromatographic system was eluted through the $n$-hexane-ethyl acetate solvent system $(85: 15,84: 16$, and $80: 20)$ resulting in six (1-6) dimeric naphthoquinones (Figure 1). These isolated compounds, namely, 5,4-dihydroxy-1-methoxy-6,6-dimethyl7,3-binaphthyl-1,4,5,8-tetraone (1), 5,8-dihydroxy-5-methoxy-6,6-dimethyl-7,3-binaphthyl-1,4,1,4-tetraone (2), 8,5,8trihydroxy-6,6-dimethyl-7,3-binaphthyl-1,4,1,4-tetraone (3), $5^{\prime}, 8^{\prime}$-dihydroxy-6,6 -dimethyl-7,3' -binaphthyl-1,4,1 ', $4^{\prime}$-tetraone (4), $5^{\prime}, 8^{\prime}$-dihydroxy-5,8-dimethoxy-6,6 $6^{\prime}$-dimethyl-7, $3^{\prime}$ -binaphthyl-1,4,1 $1^{\prime}, 4^{\prime}$-tetraone (5), and 5,8,5-trihydroxy-8methoxy-6,6-dimethyl-7,3-binaphthyl-1,4,1,4-tetraone (6), were previously reported by our group. The chemical structures of compounds 1,2, and 3 were identified by NMR data by our group [12]. The same group has elucidated the 
<smiles>COc1cc(-c2cc3c(c(O)c2C)C(=O)C=CC3=O)c(O)c2c1C(=O)C=C(C)C2=O</smiles><smiles>COc1c(C)c(C2=CC(=O)c3c(O)cc(C)c(O)c3C2=O)cc2c1C(=O)C=CC2=O</smiles><smiles>Cc1cc(O)c2c(c1O)C(=O)C(c1c(C)cc3c(c1O)C(=O)C=CC3=O)CC2=O</smiles><smiles>Cc1cc2c(cc1C1=CC(=O)c3c(O)cc(C)c(O)c3C1=O)C(=O)C=CC2=O</smiles><smiles>COc1c(C)c(C2=CC(=O)c3c(O)cc(C)c(O)c3C2=O)c(O)c2c1C(=O)C=CC2=O</smiles><smiles>COc1cc(C)c(O)c2c1C(=O)C=C(c1c(C)c(O)c3c(c1O)C(=O)C=CC3=O)C2=O</smiles>

FIgURE 1: Chemical structures of isolated active phytochemicals from Diospyros lotus.

structures of compounds 4 and 5 by using NMR data [13]. Similarly, the same group has also reported compound 6 by advanced spectroscopic analysis [14].

\subsection{Anti-inflammatory Activity}

2.4.1. Carrageenan-Induced Paw Edema. BALB/c mice weighing 25 to 30 grams were procured to assess the anti-inflammatory properties of the six (1-6) dimeric naphthoquinones isolated from roots of $D$. lotus [14]. Mice were randomly categorized into various groups depending on treatment. Purposely, Group I acted as the negative control (treated with distilled water$10 \mathrm{ml} \mathrm{kg}^{-1}$ ) and Group II acted as the positive control (intraperitoneally treated with diclofenac sodium, $5 \mathrm{mg} \mathrm{kg}^{-1}$ ), while the other groups were classified as tested groups (intraperitoneally treated with the six isolated compounds, 2.5 and $5 \mathrm{mg} \mathrm{kg}^{-1}$ ). Thirty minutes after the intraperitoneal subjection of the abovementioned treatments, $1 \%$ carrageenan $(0.05 \mathrm{ml})$ was subcutaneously injected in the right hind paw (subplantar tissues) of each mouse. Inflammation in each mouse was recorded after 1, 2, 3, 4, and 5 hours of carrageenan administration through plethysmometer (LE 7500 plan lab S.L). Antiinflammatory activity (percent inhibition) of the six isolated compounds against paw edema was obtained through the following formula:

$$
\text { Inhibition }(\%)=\left(\frac{A-B}{A}\right) \times 100 \text {, }
$$

where $A$ is the paw edema of the control group and $B$ is the paw edema of the tested group.

2.4.2. Histamine-Induced Paw Edema. Histamine-induced paw edema methods were also used to assess the antiinflammatory activity of the isolated compounds 1 and 6, according to standard methods $[14,15]$. For the induction of inflammatory paw edema, $0.1 \mathrm{ml}$ of histamine solution $(0.5 \%)$ was administered at the subplantar region. The paw volume was noted at 1,2,3, 4, and 5 hours after administering the inflammatory drug. Each group of mice was pretreated orally with compounds $1-6$ (2.5 and $\left.5 \mathrm{mg} \mathrm{kg}^{-1}\right), 1$ hour before inducing paw edema. The effect of paw edema was compared with a standard drug (loratadine) against histamine-induced edema, respectively.

2.5. Antibacterial Activity. The antibacterial activity of isolated compounds was assessed by an agar well diffusion method as per reported methods. The Mueller Hinton Agar (MHA) was used as a medium. The culture was incubated in triplicate, and the incubation was done for $24-72 \mathrm{hrs}$, at $37^{\circ} \mathrm{C}$. $0.6 \mathrm{ml}$ broth culture of the tested organism was poured onto a sterile Petri dish, and then, the sterile molten $(20 \mathrm{ml})$ was mixed. Then, $6.0 \mathrm{~mm}$ holes were bored in the prepared medium and $0.2 \mathrm{ml}$ compounds were added to each well. Streptomycin $(2 \mathrm{mg} / \mathrm{ml})$ was used as a standard antimicrobial agent. After incubation $\left(37^{\circ} \mathrm{C} / 2\right.$ hours), the diameter was 
measured in millimeters $(\mathrm{mm})$ to analyze the inhibition of microbial growth.

2.6. Toxicology Study. The acute toxicity of isolated compounds 1-6 was determined as per our previously reported method [14]. The animals were divided into six groups each comprising six mice $(n=6)$. The isolated pure compounds at doses of $5,10,20,100$, and $200 \mathrm{mg} / \mathrm{kg}$ body weight of animal were used in this study. After administration of compounds at the test doses, the animals of each group were kept under observation for $24 \mathrm{hrs}$. The number of survived and dead animals of each group was recorded, and the mortality was calculated as per standard procedure [16].

2.7. Statistical Analysis. Results of this study are stated as mean \pm SEM. To find out the significant difference $(p<0.05$ or 0.01) among the experimental groups, one-way Analysis of Variance (ANOVA) was performed followed by Dunnett's multiple comparison test.

\subsection{Computational Studies}

2.8.1. Docking Studies. Molecular operating environment (MOE 2016.08) was used for performing the docking studies [17]. Protein Data Bank (PDB; code: 1CX2) was used for the retrieval purpose of crystalline structure of COX-2 along with SC-558. For COX-1, the 3D structure was retrieved as accession number 1EQG with ibuprofen as the native ligand. Validation of the docking method was ensured through redocking of the native ligands. For ligand preparation, protonation of 3D coordinates, binding site determination, and downloaded enzymes, our previously published methods were adopted [18-20]. The builder option in a drug discovery software program, i.e., molecular operating environment, was used for drawing of ligand structures. All the database regarding tested compounds was built as ligand.mdb. Further, the MMFF94X force field was used for minimizing the energy of compounds up to 0.01. The structure of enzymes was opened in a molecular operating environment window. 3D protonation of all the atoms was achieved under solvated conditions (temperature: $300 \mathrm{~K}$; pH: 7; salt content: 0.1). The MMFF94X force field was used for minimizing the energy of the complete structure. Lastly, binding pockets of target enzymes were docked with all the compounds. After setting all the default docking parameters, 10 different conformations were produced for each compound. The MOE ligand interaction module was used to assess the least binding energy of ligand-enzyme complexes. Then, the Discovery Studio visualizer was employed for production of the $3 \mathrm{D}$ interaction plot [21].

2.8.2. In Silico Pharmacokinetic Prediction. An online tool named pkCSM was employed to predict the in silico pharmacokinetic characteristics of the isolated biomolecules [21]. SMILES (Simplified Molecular-Input Line-Entry Specification) notations were used as sequential inputs that represent a two-dimensional chemical structure through strings.

\section{Results}

3.1. Anti-inflammatory Effect. Figure 2 shows the antiinflammatory properties of different doses $\left(2.5\right.$ and $\left.5 \mathrm{mg} \mathrm{kg}^{-1}\right)$ of six (1-6) compounds isolated from roots of $D$. lotus. The maximum protection against carrageenan-induced paw edema was observed in groups of mice treated with compounds 1 and 2. Both of the tested compounds demonstrated significant antiinflammatory effect after the 3rd hour of treatment. Percent inhibition of paw edema in groups administered with compound 1 was $85.96 \%$, while in the case of groups subjected to compounds 2 , 5, and 6 , this value was $81.44 \%, 80.11 \%$, and $82.45 \%$, respectively. Furthermore, inhibitory action of these compounds against paw edema was in a concentrationdependent manner. Of all the tested compounds, 3 and 4 did not reveal any significant effect as compared to the negative control; meanwhile, the effect of the positive control (diclofenac sodium) was maximum (96.4\%) among tested samples.

3.2. Histamine-Induced Paw Edema. Histamine-induced inflammatory paw edema was significantly inhibited by compounds $1,2,5$, and 6 at $5 \mathrm{mg} / \mathrm{kg}$, i.p. The maximum effect of compound 1 was $84.98 \%$ and compound 2 was $82.18 \%$, while compounds 5 and 6 were $84.39 \%$ and $77.44 \%$, respectively, after the $2^{\text {nd }}$ hour of posttreatment in a concentrationdependent manner and remained good up to the 5th hour of administration as given in Figure 3.

3.3. Antibacterial Effect. The isolated compounds 1-6 were also screened against five Gram-positive and Gram-negative bacterial strains (Table 1). The tested compounds exhibited good activities against the Gram-positive bacteria, namely, Staphylococcus aureus, Bacillus subtilis, and Streptococcus epidermis with inhibition zones ranging from 8.0 to $22.0 \mathrm{~mm}$. On the other hand, the Gram-negative bacteria (Klebsiella pneumoniae and Escherichia coli) did not reveal any susceptibility against the tested compounds.

3.4. Acute Toxicity Effect. The acute toxicity of isolated compounds 1-6 was evaluated in the dose range of $5,10,100$, and $200 \mathrm{mg} / \mathrm{kg}$, respectively. After administering the tested doses of compounds 1-6 intraperitoneally (i.p.), the animals were kept for 2 days under observation. Then, the numbers of dead and surviving animals were calculated. All the animals survived up to the maximum tested doses. All isolated compounds were assessed for toxicity test, and interestingly, all the animals were found safe up to the maximum tested doses. No toxicity was observed for compounds 1-6; their overall acute toxicity was found safe at all test doses $(5,10$, 100 , and $200 \mathrm{mg} / \mathrm{kg}$ ) over the $24 \mathrm{~h}$ assessment period.

3.5. Docking Results. Docking studies were performed on target enzymes for the authentication of the results of in vitro experimentation, analysis of binding orientation, and ligand-enzyme interactions. In this part of the study, results of in vivo experimentation were explored. The model of carrageenan-induced rat paw edema was adopted to assess the anti-inflammatory potential of isolated compounds. This model is known as the COX-2-dependent model of inflammation and is performed to validate the anti-inflammatory 


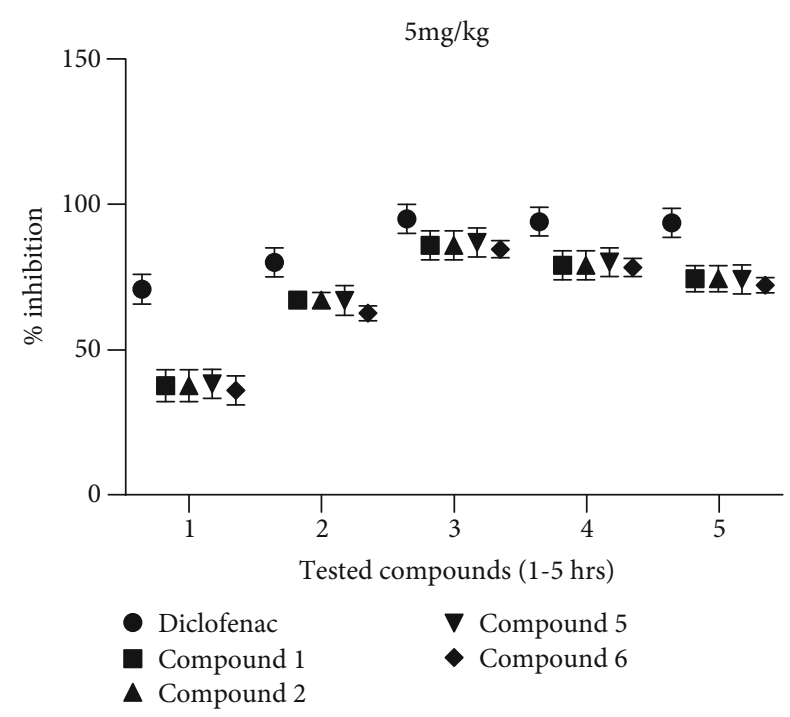

FIgURE 2: Anti-inflammatory activity of compounds 1-6 (isolated from Diospyros lotus) on carrageenan-induced paw edema in mice. Each bar shows percent inhibition of paw edema after 1, 2, 3, 4, and 5 hours of treatment. All data were analyzed by ANOVA followed by Dunnett's test.

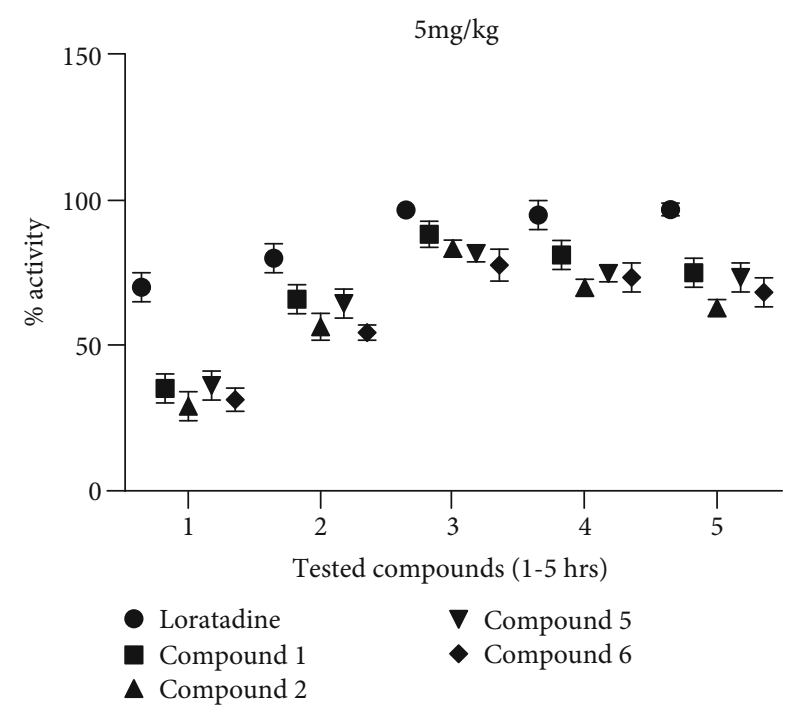

Figure 3: Anti-inflammatory activity compounds 1-6 (isolated from Diospyros lotus) on histamine-induced paw edema in mice. Each bar showed percent inhibition of paw edema after 1, 2, 3, 4, and 5 hours of treatment. All data were analyzed by ANOVA followed by Dunnett's test.

characteristics of drugs. Moreover, we used diclofenac as the standard drug in this assay. The mechanism of diclofenac for its anti-inflammatory action is the inhibition of the synthesis of prostaglandin by inhibition of the transiently expressed COX-2 isozyme. Hence, the COX-2 enzyme was used for docking simulations in this study. The molecular operating environment [17] suit was used for this purpose. All the isolated active phytochemicals (1-6) were docked at binding sites of COX-1 and COX-2 isoforms. The binding poses of the phytochemicals superposed on the native ligand S58 are shown in Figure 4. It can be observed that all these compounds occupied the binding site of the native ligand.

From analysis, the three-dimensional interaction plot of the compounds into the binding site of COX-2 showed that almost all the compounds interacted with the amino acid residues (Ser353, Leu352, Gln192, Arg513, His90, and Val523) that existed at an additional secondary site. Compound 1 established a hydrogen bond interaction (HBI) with Arg513, while the phenyl ring showed $\pi-\pi$ interactions with Tyr355 (Figure 5(a)). Compound 2 formed HBIs with Leu352, Tyr385, Gly526, and Ala527 (Figure 5(b)).

The phenyl ring showed $\pi-\pi$ interactions with Tyr355, while compound 3 showed HBIs with Arg120, Val523, and Ser530. Tyr355 formed $\pi$-lone pair interaction with the oxygen atom of the hydroxyl group (Figure 6(a)). Compound 4 also showed HBI with Tyr355, Tyr385, and Arg513. Met522 formed $\pi$-sulfur-type interactions (Figure 6(b)).

The 3D interaction plot of compounds 5 and 6 is shown in Figures 7(a) and 7(b). Compound 5 established HBIs with His90, Arg120, Tyr385, Arg513, and Glu524 (Figure 7(a)). Compound 6 formed $\pi$-lone pair interaction with Tyr355. It also showed HBIs with Arg120, Tyr385, and Trp387 (Figure $7(\mathrm{~b})$ ). The binding affinity values of all the compounds against COX-2 are shown in Table 2. Docking studies on the COX-1 isozyme were also performed using the $3 \mathrm{D}$ structure of 1EQG. The 3D interaction plots of isolated compounds 16 are shown in Figures 8(a)-8(f). Figures 8(a)-8(f) show that all the compounds had stable hydrogen bond interactions as well as $\pi-\pi$ interactions with Arg120, Tyr355, and Ser530. The binding affinity results are shown in Table 2.

3.6. In Silico Predictions of Pharmacokinetics. For the establishment of in vivo-in silico relationship, initiation of in silico pharmacokinetic prediction for all the isolated biomolecules was investigated. The main aim of this experimentation was to predict the in vivo pharmacokinetic properties of the compounds isolated (1-6) from Diospyros lotus, depending upon their virtual structure and derived parameters. For this purpose, various molecular descriptors as indicators of molecular properties (Lipinski rule of 5), absorption (water solubility, human intestinal absorption), distribution (volume of distribution, fraction of unbound, blood-brain barrier permeability, and CNS penetration), excretion (total clearance), and toxicity (hERG I and II inhibitors, AMES toxicity, and hepatotoxicity) were computed. The molecular descriptors were calculated by using the pkCSM online tool. The results are presented in Table 3 .

Almost all the phytochemicals (1-6) fulfilled the Lipinski Ro5 parameters. Data indicates that all compounds showed good human intestinal absorption (HIA, poor absorption $\leq 30 \%)$. The water solubility value is given in $\log (\mathrm{mol} / \mathrm{l})$. The value of solubility $<-10$ indicates that the substance is insoluble. Highly soluble compounds showed values $<0$. For the distribution of the drug in the brain, compounds having $\log \mathrm{BB}$ values less than 0.3 are able to cross the $\mathrm{BBB}$. Meanwhile, compounds having $\log \mathrm{BB}$ values less than -1 are not considered to cross BBB easily. The distribution of the drug in various tissues is measured by the 
Table 1: Antibacterial activity of compounds 1-6 isolated from Diospyros lotus.

\begin{tabular}{lcccccccc}
\hline \multirow{2}{*}{ Bacterial strains } & Control & 1 & \multicolumn{4}{c}{ Inhibitory zone (mm) } & \multicolumn{2}{c}{ Streptomycin (2 mg/ml) } \\
\hline Escherichia coli & 0 & 0 & 0 & 0 & 0 & 0 & 0 & $32 \pm 0.55$ \\
Staphylococcus aureus & 0 & $14 \pm 0.83$ & $12 \pm 0.97$ & $16 \pm 0.81$ & $13 \pm 0.66$ & $18 \pm 0.89$ & $14 \pm 0.22$ & $31 \pm 0.66$ \\
Bacillus subtilis & 0 & $10 \pm 0.42$ & $11 \pm 0.66$ & $08 \pm 0.87$ & $08 \pm 0.33$ & $15 \pm 0.97$ & $16 \pm 0.99$ & $33 \pm 0.44$ \\
Klebsiella pneumoniae & 0 & 0 & 0 & 0 & 0 & 0 & 0 & $30 \pm 0.99$ \\
Streptococcus epidermis & 0 & $19 \pm 0.87$ & $13 \pm 0.55$ & $10 \pm 0.11$ & $13 \pm 0.78$ & $18 \pm 0.98$ & $22 \pm 0.98$ & $32 \pm 0.43$ \\
\hline
\end{tabular}
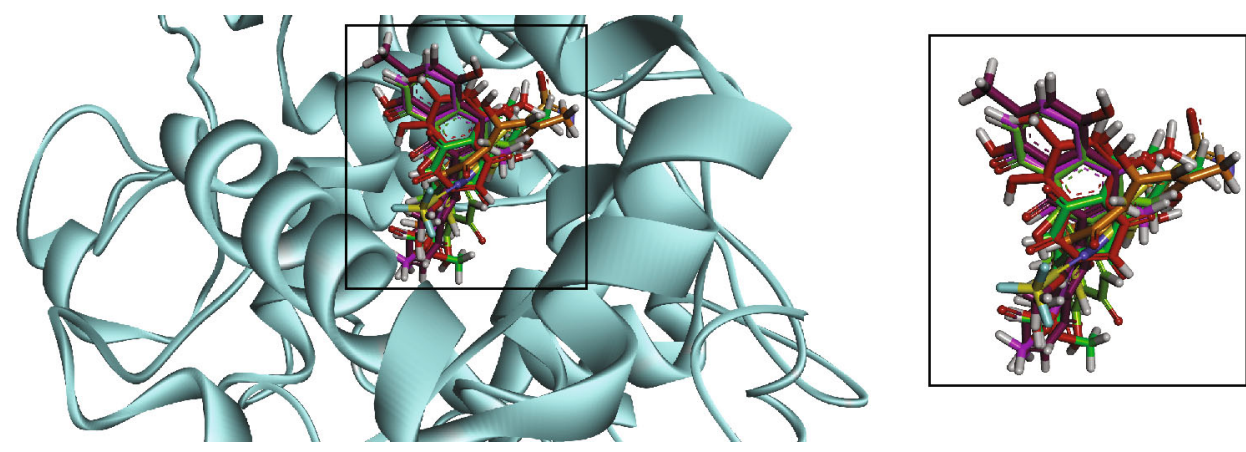

Figure 4: Ribbon model of the superimposed binding poses of compounds 1-6 on the native drug (yellow) into the binding site of human COX-2 (1CX2).

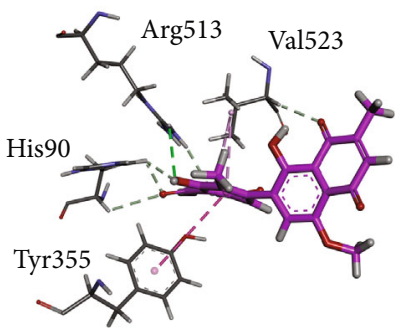

(a)

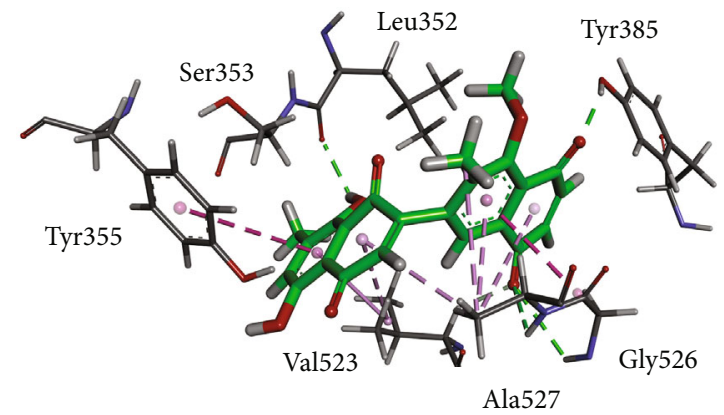

(b)

FIgURE 5: (a, b) Close-up 3D interaction plot of compounds 1 and 2 into the binding site of human COX-2 (1CX2).

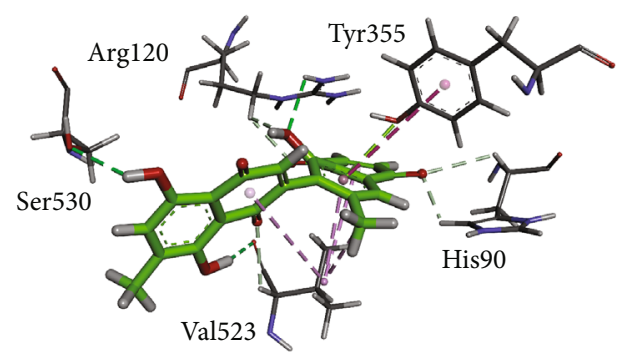

(a)

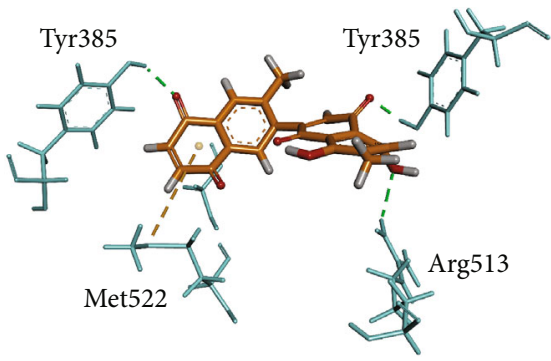

(b)

FIgUre 6: ( $\mathrm{a}, \mathrm{b})$ Close-up 3D interaction plot of compounds 3 and 4 into the binding site of human COX-2 (1CX2).

volume of distribution (VD). Here, VD is considered to be lower when the value of log VDss is less than -0.15 and high when it is greater than 0.45 . In our case, almost all compounds, except 4 , showed high volume of distribution. The elimination of the drug from the body is mainly associated with hydrophilicity and molecular weight of the 


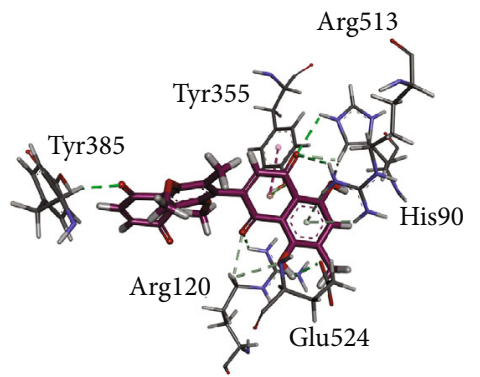

(a)

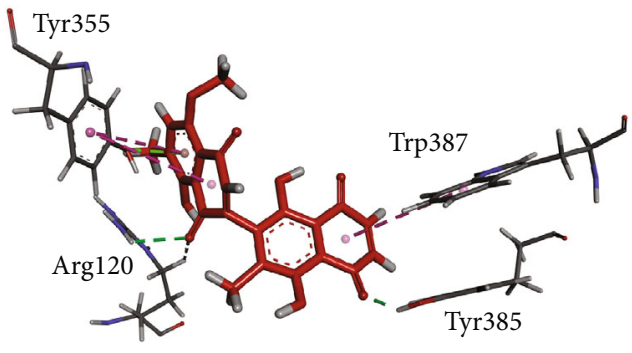

(b)

Figure 7: (a, b) Close-up 3D interaction plot of compounds 5 and 6 into the binding site of human COX-2 (1CX2).

TABLE 2: Binding affinity values computed via MOE software for COX-1 and COX-2 inhibition.

\begin{tabular}{lcc}
\hline \multirow{2}{*}{ Compound } & \multicolumn{2}{c}{ Binding affinity $(\mathrm{kcal} / \mathrm{mol})$} \\
& COX-1 & COX-2 \\
\hline 1 & -3.6128 & 6.6910 \\
2 & -2.5655 & -6.6744 \\
3 & -3.4539 & -5.8738 \\
4 & -3.7816 & -5.1819 \\
5 & -2.3318 & -6.3898 \\
6 & -2.5912 & -6.0694 \\
\hline
\end{tabular}

drug. The computed results (in $\log \mathrm{ml} / \mathrm{min} / \mathrm{kg}$ ) shown in Table 3 revealed that compound 1 has the highest clearance rate. The order of the clearance for other compounds is $5>6>2>4>3$. The toxicity of the compounds was predicted in terms of hERG channel inhibition (cardiotoxicity), AMES, and hepatotoxicity. The results shown in Table 3 revealed that all the 6 compounds might not have inhibitory action on the hERG channel. Therefore, these compounds might not pose cardiotoxicity.

\section{Discussion}

Inflammation is a painful edematous condition associated with various disorders. Prostaglandins (PGE2 and PGE2 $\alpha$ ) are considered to be responsible for inflammation due to increased capillary permeability. COX-1 and COX-2 are the enzymes that produce these prostaglandins (PGs). Antiinflammatory drugs (nonsteroidal) prescribed during inflammation and available in the market actually block the activity of these enzymes (COX-1 and COX-2), hence resulting in reduction of inflammation. In traditional medicine, plants comprising naphthoquinones are investigated against inflammation and cancer to function as sedative and analgesic [22]. However, under chronic inflammatory conditions, these drugs are administrated for a longer period of time. This prolonged exposure could lead to various gastrointestinal tract problems like ulcer. Therefore, it is a challenging task for modern-day medicinal chemists and pharmacists to discover safe and effective molecules having the least adverse effects. Interestingly, the compounds 1-6 were not toxic at test doses. Diospyros lotus is traditionally used in different inflammatory conditions. The chloroform fraction of D. lotus has been documented for significant anti-inflammatory action [23]. The current compounds were isolated from the chloroform fraction of Diospyros lotus and tested for the same effect in order to investigate the chemical constituents of this fraction as anti-inflammatory agents. The antibacterial evaluation showed that the extracted compounds are most effective against the Gram-positive bacteria. In fact, the Gram-negative bacteria are considered difficult-to-treat microorganisms, due to the nature of their outer membrane which protects them from lethal effects of antibacterial agents [24]. However, the noticeable inhibition effect of the extracted compounds against the Gram-negative bacteria requires further antibacterial investigation to understand the minimum inhibitory bactericidal concentrations and the mode of action. Docking results revealed that almost all the compounds showed good interactions with the amino acid residues of the additional pocket present in the COX-2 isozyme. Hence, in the absence of in vitro results, we suggest that these compounds may be selective COX-2 inhibitors. This was also confirmed by the computed binding affinity data. All compounds showed more negative binding affinity for COX-2 inhibition than COX-1. Drug likeness of the compounds was predicted by using the online pkCSM tool. Almost all the results are within the acceptable ADMET range. The Lipinski rule was followed by all the isolated compounds. Steady-state volume of distribution (VDss) was estimated and revealed that almost all the compounds isolated may be distributed evenly. The crossing of the $\mathrm{BBB}$ by exogenous compounds 1-6 may cause side effects and toxicity. However, some neurological disorders like Alzheimer's disease $(\mathrm{AD})$ may require the drug to cross $\mathrm{BBB}$. The $\mathrm{BBB}$ permeability was predicted in a study as $\operatorname{logBB}$. The negative values showed that all the isolated compounds 1-6 cannot cross the BBB. As such, it can be concluded that these drugs may not be used against neuroinflammation. The toxicity of the isolated compounds in this study was predicted in terms of hERG inhibition, AMES toxicity, and hepatotoxicity. As far as cardiotoxicity is concerned, inhibition of the human ether-a-go-go-related gene (hERG) channel also has great effects on human health. Results in the current screening exhibited that all the tested compounds 16 were safe and possessed no cardiotoxicity. Moreover, these compounds were predicted to have no hepatotoxicity and to be non-AMES toxic $[25,26]$. 


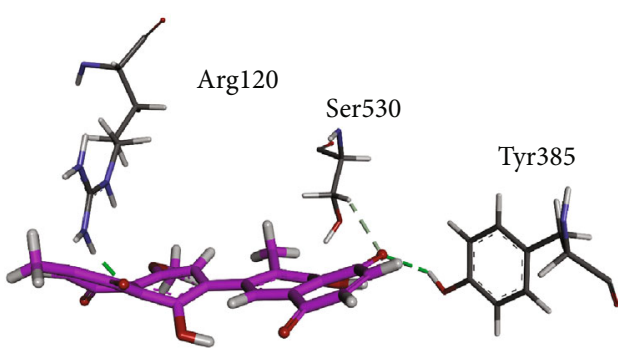

(a)

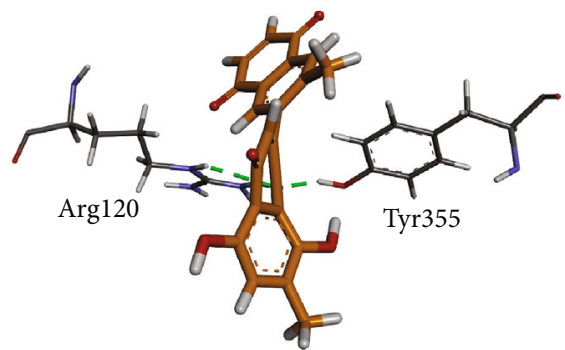

(d)

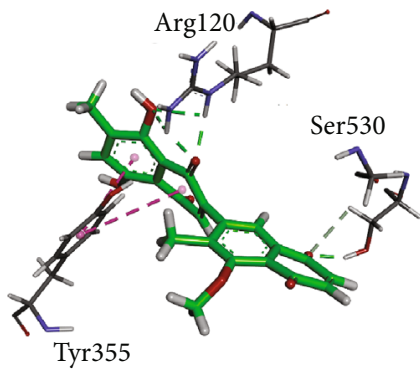

(b)

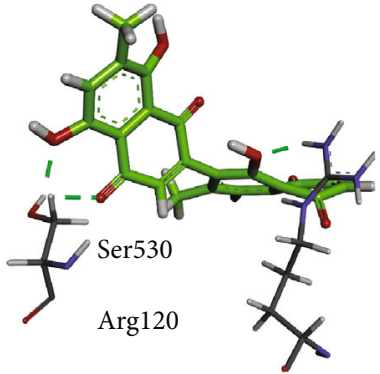

(c)

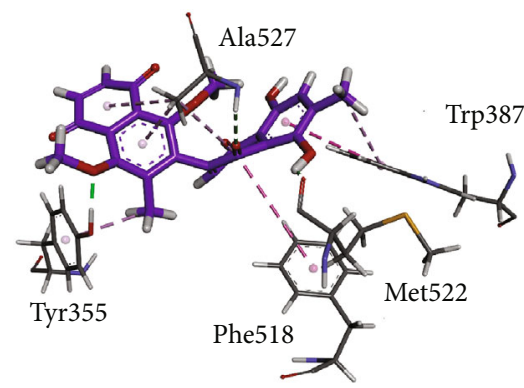

(e)

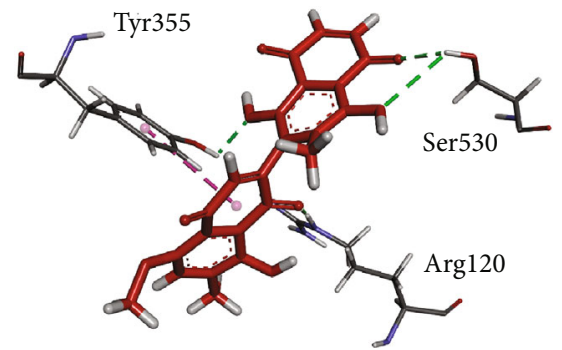

(f)

FIgURE 8: (a-f) Close-up 3D interaction plot of compounds 1-6 into the binding site of human COX-1 (1EQG).

TABLE 3: In silico pharmacokinetic predictions of the isolated phytochemicals.

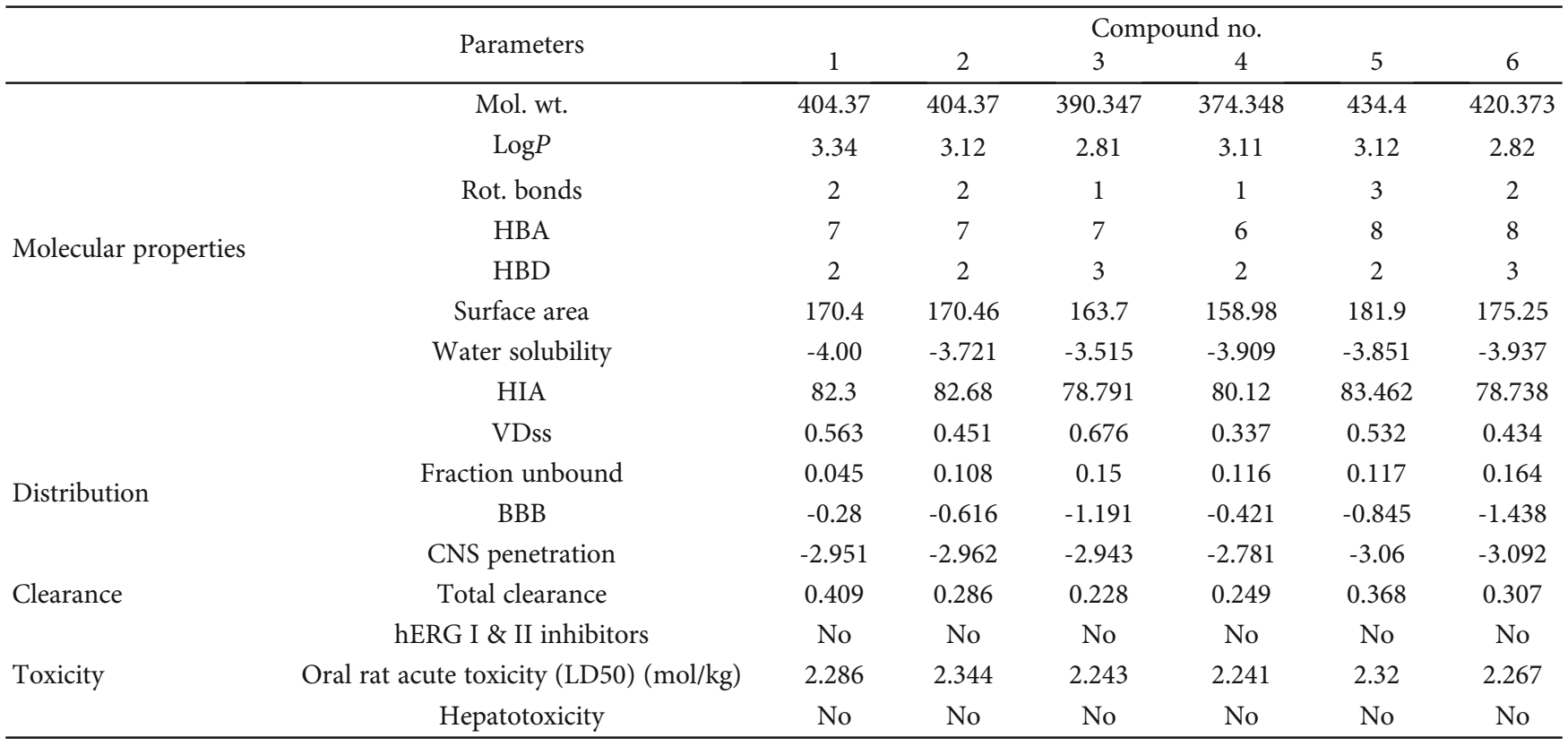

\section{Conclusions}

This study has revealed that compound 1, 2, 5, and 6 exhibited significant anti-inflammatory properties in carrageenan-induced paw edema among all the six (1-6) compounds isolated from roots of Diospyros lotus. Results of this study could provide a baseline data for pharmacological use of these isolated compounds as anti-inflammatory agents. All isolated compounds were also assessed for anti- bacterial sensitivity, among which compounds 1-6 exhibited remarkable activity against Staphylococcus aureus, Bacillus subtilis, and Streptococcus epidermis. Docking studies on the COX-2 enzyme were also performed. In the absence of in vitro results, it is suggested that these compounds may be selective COX-2 inhibitors. Drug likeness of the compounds was predicted by using the online pkCSM tool. Almost all the results obtained are within the acceptable drug-like properties. 


\section{Abbreviations}

AD: $\quad$ Alzheimer's disease

ADMET: Absorption, distribution, metabolism, excretion, and toxicity

BBB: Blood-brain barrier

COX-1: Cyclooxygenase-1

COX-2: Cyclooxygenase-2

hERG: Human ether-a-go-go-related gene

HIA: Human intestinal absorption

i.p.: Intraperitoneally

MMFF: $\quad$ Merck molecular force field

MOE: Molecular operating environment

PDB: $\quad$ Protein Data Bank

PGs: $\quad$ Prostaglandins

pkCSM: Predicting Small-Molecule Pharmacokinetic and Toxicity Properties

VD: $\quad$ Volume of distribution

VDss: Steady-state volume of distribution.

\section{Data Availability}

The data such as spectra and associated analysis used to support the finding of this study are available from the corresponding author upon request.

\section{Conflicts of Interest}

The authors declare no potential conflict of interest.

\section{Acknowledgments}

The authors are grateful to Higher Education Commission (HEC) of Pakistan. Also, the authors acknowledge the Deanship of Scientific Research at King Khalid University for funding this prolific research group no. R.G.P.2.115/41.

\section{References}

[1] G. Uddin, A. Rauf, and M. Arfan, "Molecular docking of diospyrin as a LOX inhibitory compound," Journal of Saudi Chemical Society, vol. 20, pp. 448-450, 2016.

[2] G. Uddin, A. Rauf, B. S. Siddiqui, and S. Q. Shah, "Preliminary comparative phytochemical screening of Diospyros lotus Stewart," Middle-East Journal of Scientific Research, vol. 10, pp. 78-81, 2011.

[3] G. Uddin, A. Rauf, B. S. Siddiqui, N. Muhammad, A. Khan, and S. U. A. Shah, "Anti-nociceptive, anti-inflammatory and sedative activities of the extracts and chemical constituents of Diospyros lotus L.," Phytomedicine, vol. 21, no. 7, pp. 954959, 2014.

[4] M. Tezuka, C. Takahashi, M. Kuroyanagi, M. Satake, K. Yoshihira, and S. Natori, "New naphthoquinones from Diospyros," Phytochemistry, vol. 12, no. 1, pp. 175-183, 1973.

[5] S. Ganapaty, P. Steve Thomas, G. Karagianis, P. G. Waterman, and R. Brun, "Antiprotozoal and cytotoxic naphthalene derivatives from Diospyros assimilis," Phytochemistry, vol. 67, no. 17, pp. 1950-1956, 2006.

[6] M. R. Loizzo, A. Said, R. Tundis et al., "Antioxidant and antiproliferative activity of Diospyros lotus L. extract and isolated compounds," Plant Foods for Human Nutrition, vol. 64, no. 4, pp. 264-270, 2009.

[7] R. Verma, "Anti-cancer activities of 1, 4-naphthoquinones: a QSAR study," Anticancer Agents in Medicinal Chemistry, vol. 6, no. 5, pp. 489-499, 2006.

[8] S. Padhye, P. Dandawate, M. Yusufi, A. Ahmad, and F. H. Sarkar, "Perspectives on medicinal properties of plumbagin and its analogs," Medicinal Research Reviews, vol. 32, no. 6, pp. 1131-1158, 2012.

[9] A. Rauf, G. Uddin, B. S. Siddiqui, N. Muhammad, and H. Khan, "Antipyretic and antinociceptive activity of Diospyros lotus L. in animals," Asian Pacific Journal of Tropical Biomedicine, vol. 4, Suppl 1, pp. S382-S386, 2014.

[10] S. Bawazeer, A. Rauf, S. U. Shah et al., "Antioxidant and enzyme inhibitory activities of extracts and phytochemicals isolated from Pistacia integerrima," Journal of Medicinal and Spices Plants, vol. 24, pp. 55-58, 2019.

[11] A. Rauf, G. Uddin, N. Jehan et al., "Fatty acids profile, squalene level and biological traits of lipids from Diospyros lotus roots," Journal of Medicinal Spices and Plants, vol. 22, pp. 84-87, 2017.

[12] A. Rauf, G. Uddin, B. S. Siddiqui et al., "A Rare class of new dimeric Naphthoquinones from Diospyros lotus have multidrug reversal and antiproliferative effects," Frontiers in Pharmacology, vol. 6, p. 293, 2015.

[13] A. Rauf, G. Uddin, B. S. Siddiqui et al., "Bioassay-guided isolation of novel and selective urease inhibitors from Diospyros lotus," Chinese Journal of Natural Medicines, vol. 15, no. 11, pp. 865-870, 2017.

[14] A. Rauf, T. B. Hadda, S. Patel et al., "Identification, structure elucidation, and antioxidant potential of a new compound from Diospyros lotus," Chemistry of Natural Compounds, vol. 53, no. 5, pp. 849-851, 2017.

[15] N. Muhammad, M. Saeed, and H. Khan, “Antipyretic, analgesic and anti-inflammatory activity of Viola betonicifolia whole plant," BMC Complementary Alternative Medicine, vol. 12, pp. 59-61, 2012.

[16] H. Khan, M. Saeed, A. U. H. Gilani, M. A. Khan, A. Dar, and I. Khan, "The antinociceptive activity of Polygonatum verticillatum rhizomes in pain models," Journal of Ethnopharmacology, vol. 127, no. 2, pp. 521-527, 2010.

[17] A. Rauf, G. Uddin, B. S. Siddiqui, and H. Khan, "In vivo sedative and muscle relaxants activity of Diospyros lotus L," Asian Pacific Journal of Tropical Biomedicine, vol. 5, no. 4, pp. 277280, 2015.

[18] Molecular Operating Environment (MOE), 2016.08; Chemical Computing Group ULC, 1010 Sherbooke St. West, Suite \#910, Montreal, QC, Canada, H3A 2R7, 2018.

[19] F. Iftikhar, F. Yaqoob, N. Tabassum et al., "Design, synthesis, in-vitro thymidine phosphorylase inhibition, in-vivo antiangiogenic and in-silico studies of C-6 substituted dihydropyrimidines," Bioorganic Chemistry, vol. 80, pp. 99-111, 2018.

[20] M. S. Jan, S. Ahmad, F. Hussain et al., "Design, synthesis, invitro, in-vivo and in-silico studies of pyrrolidine-2, 5-dione derivatives as multitarget anti-inflammatory agents," European Journal of Medicinal Chemistry, vol. 186, p. 111863, 2020.

[21] S. T. Tanoli, M. Ramzan, A. Hassan et al., "Design, synthesis and bioevaluation of tricyclic fused ring system as dual binding site acetylcholinesterase inhibitors," Bioorganic Chemistry, vol. 83, pp. 336-347, 2019. 
[22] D. S. Biovia, Discovery Studio visualizer, San Diego, CA, USA, 2017.

[23] A. Rauf, G. Uddin, and B. S. Siddiqui, "Isolation and structure elucidation of a new dimeric Naphthoquinone from Diospyros lotus," Chemistry of Natural Compounds, vol. 51, no. 6, pp. 1049-1051, 2015.

[24] S. I. Miller, "Antibiotic resistance and regulation of the Gramnegative bacterial outer membrane barrier by host innate immune molecules," MBio, vol. 7, no. 5, p. e01541, 2016.

[25] A. A. Elhenawy, L. M. al-Harbi, M. A. el-Gazzar et al., "Naproxenylamino acid derivatives: Design, synthesis, docking, QSAR and anti- inflammatory and analgesic activity," Biomedicine and Pharmacotherapy, vol. 116, p. 109024, 2019.

[26] Y. Han, J. Zhang, C. Q. Hu, X. Zhang, B. Ma, and P. Zhang, "In silico ADME and toxicity prediction of ceftazidime and its impurities," Frontiers in Pharmacology, vol. 10, p. 434, 2019. 